\title{
Progressive Educational and Developmental Psychology
}

In this issue, the focus of psychological issues in schools is the predominant feature. There are six articles covering various themes that are designed to enhance practice and research knowledge in educational and developmental psychology. The journal is attempting to regularly increase the number of articles per issue from five to at least six, and this is the second issue in a row where this has been achieved. There has been an increase in submissions to the journal, but the quality of the articles that are accepted for publication still remains very high.

In the first article, authors Hall and Lindorff investigate the issue of stress related to children transitioning from preschool to primary school. The authors discuss the issue of school readiness and the importance of ensuring the continuity of routines during this transition process. The next article, authored by Bosnjak et al., discusses a pilot study in secondary schools that utilised cognitive behavioural therapy principles to improve students' attributions for success and failure in learning. The results from the study demonstrated the potential for attribution retraining with secondary school students. In the third article of this issue, Allen et al.'s school study demonstrates a link between academic motivation, school belonging, and mental health promotion. The researchers found that the latter activity in a school can lead to success in the other areas of the study and thus can lead to improved motivation to achieve academically.

Ethical challenges in school psychology are explored in the fourth article of this issue. Kapoulitsas and Corcoran explore the issues in this context, which highlight the importance of peer support and provide suggestions for policy development to enhance ethical knowledge in workplace situations. In article five Heaslip and Barber put forward findings that highlight the importance of recognising that risk-taking behaviour is a necessary part of adolescence. Their study suggests that a positive, controlled environment is essential to reduce the potential of harm in some adolescents. In the final issue this month, Page and Charteris put forward an alternative view of relational aggression in females and suggest that it should be regarded as a normal set of behaviours that are related to communication strategies.

This issue is the final one under my editorship, as my five-year tenure ends after this set of papers. It has been a pleasure to work with so many talented authors and psychologists in Australia and elsewhere. The support of the College of Educational and Developmental Psychologists has been invaluable. The journal is now in a healthy position and can be found in all the main international research indices. The Educational and Developmental Psychologist has become a national and internationally respected journal that is distributed by one of the world's leading publishing houses. It is testament to the quality of this journal that so many high quality authors have seen their work successfully displayed on these pages. I hope my five years of 
editorship have advanced the journal and contributed to psychological knowledge for practitioner and research psychologists alike.

On a personal note, I would like to put on record my sincere thanks to Kelly Allen, Tim Corcoran, Jake Kraska, and Shane Costello, among many others who have given their valuable advice and time in various roles associated with this journal.

Christopher Boyle, $\mathrm{PhD}$

Editor 